Pulsars : Problems \& Progress

ASP Conference Series, Vol. 105, 1996

S. Johnston, M. A. Walker and M. Bailes, eds.

\title{
Pulsar Astrometry and Improved Terrestrial Clocks
}

\author{
Demetrios N. Matsakis and Frederick J. Josties \\ U.S. Naval Observatory, Washington, DC 20392
}

Roger S. Foster

Code 7213, Naval Research Laboratory, Washington, DC 20375

\begin{abstract}
Recent improvements in cesium and hydrogen terrestrial frequency standards have brought the frequency precision of International Atomic Time (TAI) to a value of $2.5 \mathrm{E}-15 \mathrm{~s} / \mathrm{s}$ over an averaging time of a month. In this paper we illustrate the improvement graphically, and discuss the state of the art for frame ties between the radio, dynamical, and optical frames. In a larger paper, available via the World Wide Web, we illustrate the measured accuracy curves of the frequency standards, show their effect on the ensemble time scales, explain the reasons for the confusing array of available time scales, and discuss the inverse problem of using pulsar data to correct the terrestrial time scale.
\end{abstract}

\section{Introduction}

In the early 1990's, terrestrial time scales, such as International Atomic Time (TAI), improved dramatically due to improved quality, quantity, and environmental isolation of frequency standards. A slightly over-dramatic description of this is given in Figure 1, which shows the frequency difference between the U.S. Naval Observatory's free-running time scale A.1 and TAI. The improvement in recent years is due to the improvement in TAI, improvements at the USNO, and the approximately $40 \%$ weight assigned to USNO clocks in the computation of TAI, which also includes about 150 other clocks maintained by approximately 50 institutions.

Because of limited space, we must refer the discussion of how terrestrial time scales are generated, and how they affect analyses involving pulsar timing, to Matsakis and Foster (1996), which is also available on-line via the World Wide Web at http://orion.usno.navy.mil/dnm.html.

\section{Frame Ties}

Ties between the radio, optical, and planetary reference frames have also improved recently. While the FK5 (optical) and IERS (radio) frames are tied together by a rotation known to an accuracy of 200 mas, the optical Hipparcos observations promise to be as close as $0.5+0.5\left(t_{\text {years }}-1991.25\right)$ mas to the radio frame, where the time-dependent part is due to proper motion errors (Lin- 
degren and Kovalevsky 1995). Similarly, the best ties between the planetary (DE403) and the radio frames are accurate to approximately 3 mas (Standish 1995, Folkner et al. 1994).

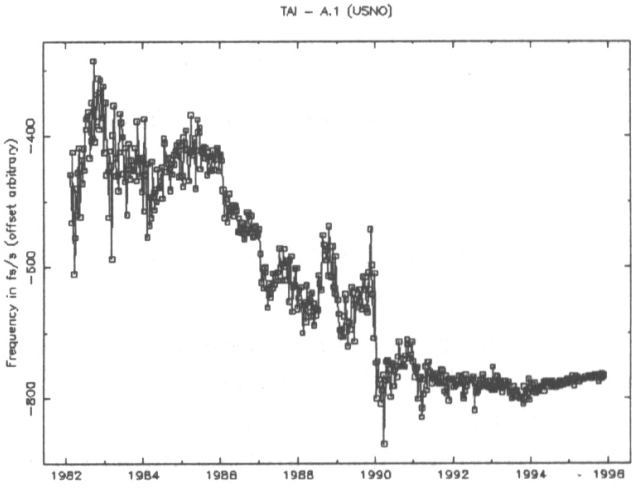

Ties between the pulsar and radio frames have been established using phasereferenced VLBI, and are accurate to a few mas (Dewey \& Beasley, this volume). Since a one microsecond pulsar timing error corresponds to a dynamical error of 0.4 mas (ignoring projection effects), the pulsar frame ties are not significantly better than the existing state of the art. However, modern frame ties are epochdependent, and the planetary frame is based upon data taken within the ecliptic plane, which limits the sensitivity to out of plane (inclination) errors. Pulsars may be able to usefully constrain the frame ties because they mostly involve observations off the ecliptic. Due to their long observational history, they may also be able to usefully constrain the time dependence of the frame tie.

Formal errors for Earth's orbit are based upon Viking data, which have an internal error of 7 meters, or 0.023 microseconds. For the Earth's orbit in 1979.6, this translates into internal orbital errors of order 0.2 mas for the Earth's perihelion and anomaly, and pulsar timing errors of order .03 microseconds (Standish 1990). If the "Mars Pathfinder" mission, scheduled to be launched in 1996, is unsuccessful these errors will roughly triple by the year 2000 .

Although pulsar timing data may seem too coarse to contribute to the frame ties, the quoted ephemeris errors are probably too optimistic. They would be incorrect, for example, if unknown objects are perturbing the solar system.

\section{References}

Folkner, W.M., Charlot, P., Finger, M.H., Williams, J.G., Sovers, O.J., Newhall, X.X., Standish, E.M., Jr 1994, A\&A, 287, 279.

Lindegren, L., Kovalevsky, J, 1995, A\&A, 304, 189.

Matsakis, D.N., and Foster, R. S. 1996, Amazing Light, a Festchrift in Honor of Charles H. Townes, Springer-Verlag Press

Standish, E.M., Jr, 1990, A\&A, 233, 252

Standish, E.M., Jr, 1995, JPL IOM 314.10-124 